\title{
Explicit Approximate Model Predictive Control of Constrained Nonlinear Systems with Quantized Input
}

\author{
Alexandra Grancharova ${ }^{\star}$ and Tor A. Johansen ${ }^{\star \star}$ \\ * Institute of Control and System Research, Bulgarian Academy of Sciences, \\ P.O.Box 79, Sofia 1113, Bulgaria, alexandra.grancharova@abv.bg

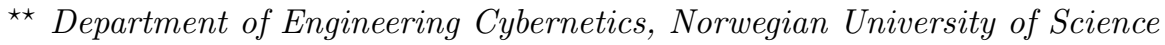 \\ and Technology, Trondheim, Norway, Tor.Arne.Johansen@itk.ntnu.no
}

Keywords : MPC, multi-parametric Nonlinear Integer Programming

\begin{abstract}
In this paper, a Model Predictive Control problem for constrained nonlinear systems with quantized input is formulated and represented as a multiparametric Nonlinear Integer Programming (mp-NIP) problem. Then, a computational method for explicit approximate solution of the resulting mp-NIP problem is suggested. The proposed approximate mp-NIP approach is applied to the design of an explicit approximate MPC controller for a clutch actuator with on/off valves.
\end{abstract}

\section{Introduction}

In several control engineering problems, the system to be controlled is characterized by a finite set of possible control actions. Such systems are referred to as systems with quantized control input and the possible values of the input represent the levels of quantization. For example, hydraulic systems using on/off valves are systems with quantized input. In order to achieve a high quality of the control system performance it would be necessary to take into account the effect of the control input quantization. Thus, in [8] receding horizon optimal control ideas were proposed for synthesizing quantized control laws for linear systems with quantized inputs and quadratic optimality criteria. Further in [1], a method for explicit solution of optimal control problems with quantized control input was developed. It is based on solving multi-parametric Nonlinear Integer Programming (mp-NIP) problems, where the cost function and the constraints depend linearly on the vector of parameters. In this paper, a Model Predictive Control (MPC) problem for constrained nonlinear systems with quantized input is formulated and represented as an mp-NIP problem. Then, a computational method for explicit approximate solution of the resulting mp-NIP problem is suggested. The benefits of the explicit solution consist in efficient on-line computations using a binary search tree and verifiability of the design and implementation. The mp-NIP method proposed here is more general compared to the mp-NIP method in [1], since it allows the cost function and the constraints to depend nonlinearly on the vector of parameters.

In the paper, $A \succ 0$ means that the square matrix $A$ is positive definite. For $x \in \mathbb{R}^{n}$, the Euclidean norm is $\|x\|=\sqrt{x^{T} x}$ and the weighted norm is defined for some symmetric matrix $A \succ 0$ as $\|x\|_{A}=\sqrt{x^{T} A x}$.

Int. Workshop on Assessment and Future Directions of NMPC

Pavia, Italy, September 5-9, 2008 


\section{Formulation of quantized Nonlinear Model Predictive Control problem}

Consider the discrete-time nonlinear system:

$$
\begin{aligned}
& x(t+1)=f(x(t), u(t)) \\
& y(t)=C x(t),
\end{aligned}
$$

where $x(t) \in \mathbb{R}^{n}$ is the state variable, $y(t) \in \mathbb{R}^{p}$ is the output variable, and $u(t) \in \mathbb{R}^{m}$ is the control input, which is constrained to belong to the finite set of values $U^{A}=\left\{\bar{u}_{1}, \bar{u}_{2}, \ldots, \bar{u}_{L}\right\}, \bar{u}_{i} \in \mathbb{R}^{m}, \forall i=1,2, \ldots, L$, i.e. $u \in U^{A}$. Here, $\bar{u}_{1}, \bar{u}_{2}, \ldots, \bar{u}_{L}$ represent the levels of quantization of the control input $u$. In (1), $f: \mathbb{R}^{n} \times U^{A} \longmapsto \mathbb{R}^{n}$ is a nonlinear function.

We consider a reference tracking problem where the goal is to have the output variable $y(t)$ track the reference signal $r(t) \in \mathbb{R}^{p}$. Suppose that a full measurement of the state $x(t)$ is available at the current time $t$. For the current $x(t)$, the reference tracking quantized NMPC solves the following optimization problem:

\section{Problem P1:}

$$
\begin{aligned}
& V^{*}(x(t), r(t))=\min _{U \in U^{B}} J(U, x(t), r(t)) \\
& \text { subject to } x_{t \mid t}=x(t) \text { and: } \\
& y_{\min } \leq y_{t+k \mid t} \leq y_{\max }, k=1, \ldots, N \\
& u_{t+k} \in U^{A}=\left\{\bar{u}_{1}, \bar{u}_{2}, \ldots, \bar{u}_{L}\right\}, k=0,1, \ldots, N-1 \\
& \left\|y_{t+N \mid t}-r(t)\right\| \leq \delta \\
& x_{t+k+1 \mid t}=f\left(x_{t+k \mid t}, u_{t+k}\right), k \geq 0 \\
& y_{t+k \mid t}=C x_{t+k \mid t}, k \geq 0
\end{aligned}
$$

Here, $U=\left[u_{t}, u_{t+1}, \ldots, u_{t+N-1}\right] \in \mathbb{R}^{N m}$ is the set of free control moves, $U^{B}=$ $\left(U^{A}\right)^{N}=U^{A} \times \ldots \times U^{A}$ and the cost function is given by:

$$
\begin{gathered}
J(U, x(t), r(t))=\sum_{k=0}^{N-1}\left[\left\|y_{t+k \mid t}-r(t)\right\|_{Q}^{2}+\left\|h\left(x_{t+k \mid t}, u_{t+k}\right)\right\|_{R}^{2}\right] \\
+\left\|y_{t+N \mid t}-r(t)\right\|_{P}^{2}
\end{gathered}
$$

Here, $N$ is a finite horizon and $h: \mathbb{R}^{n} \times U^{A} \longmapsto \mathbb{R}^{s}$ is a nonlinear function. It is assumed that $P, Q, R \succ 0$. From a stability point of view it is desirable to choose $\delta$ in (6) as small as possible. However, in the case of quantized input, the equilibrium point of the closed-loop system may either have an offset from the reference, or there may be a limit cycle about the reference. Therefore, the feasibility of (3)-(9) will rely on $\delta$ being sufficiently large. A part of the NMPC design will be to address this tradeoff. We introduce an extended state vector:

$$
\tilde{x}(t)=[x(t), r(t)] \in \mathbb{R}^{\tilde{n}}, \tilde{n}=n+p
$$

Let $\tilde{x}$ be the value of the extended state at the current time $t$. Then, the optimization problem $\mathrm{P} 1$ can be formulated in a compact form as follows:

Problem P2:

$$
V^{*}(\tilde{x})=\min _{U \in U^{B}} J(U, \tilde{x}) \quad \text { subject to } G(U, \tilde{x}) \leq 0
$$

Int. Workshop on Assessment and Future Directions of NMPC 
The quantized NMPC problem defines a multi-parametric Nonlinear Integer Programming problem (mp-NIP), since it is a Nonlinear Integer Programming problem in $U$ parameterized by $\tilde{x}$. An optimal solution to this problem is denoted $U^{*}=\left[u_{t}^{*}, u_{t+1}^{*}, \ldots, u_{t+N-1}^{*}\right]$ and the control input is chosen according to the receding horizon policy $u(t)=u_{t}^{*}$. Define the set of $N$-step feasible initial states as follows:

$$
X_{f}=\left\{\tilde{x} \in \mathbb{R}^{\tilde{n}} \mid G(U, \tilde{x}) \leq 0 \text { for some } U \in U^{B}\right\}
$$

If $\delta$ in (6) is chosen such that the problem $\mathrm{P} 1$ is feasible, then $X_{f}$ is a non-empty set.

In parametric programming problems one seeks the solution $U^{*}(\tilde{x})$ as an explicit function of the parameters $\tilde{x}$ in some set $\underline{X} \subseteq X_{f} \subseteq \mathbb{R}^{\tilde{n}}[2]$. In this paper we suggest a computational method for constructing an explicit piecewise constant (PWC) approximate solution of the reference tracking quantized NMPC problem.

\section{Approximate mp-NIP approach to explicit quantized NMPC}

\subsection{Computation of feasible PWC solution}

\section{Definition 1 (Feasibility on a discrete set):}

Let $\bar{X} \subset \mathbb{R}^{\tilde{n}}$ be a hyper-rectangle and $V_{\bar{X}}=\left\{v_{1}, v_{2}, \ldots, v_{Q}\right\} \subset \bar{X}$ be a discrete set. A function $U(\tilde{x})$ is feasible on $V_{\bar{X}}$ if $G\left(U\left(v_{i}\right), v_{i}\right) \leq 0, i \in\{1,2, \ldots, Q\}$.

We restrict our attention to a hyper-rectangle $X \subset \mathbb{R}^{\tilde{n}}$ where we seek to approximate the optimal solution $U^{*}(\tilde{x})$ to problem $\mathrm{P} 2$. We require that the state space partition is orthogonal and can be represented as a $k-d$ tree. The main idea of the approximate mp-NIP approach is to construct a feasible on a discrete set piecewise constant (PWC) approximation $\widehat{U}(\tilde{x})$ to $U^{*}(\tilde{x})$ on $X$, where the constituent constant functions are defined on hyper-rectangles covering $X$. The solution of problem P2 is computed at the $2^{\tilde{n}}$ vertices of a considered hyper-rectangle $X_{0}$, as well as at some interior points. These additional points represent the vertices and the facets centers of one or more hyper-rectangles contained in the interior of $X_{0}$. The following procedure is used to generate a set of points $V_{0}=\left\{v_{0}, v_{1}, v_{2}, \ldots, v_{N_{1}}\right\}$ associated to a hyper-rectangle $X_{0}$ :

Procedure 1 (Generation of set of points):

Consider any hyper-rectangle $X_{0} \subseteq X$ with vertices $\Lambda^{0}=\left\{\lambda_{1}^{0}, \lambda_{2}^{0}, \ldots, \lambda_{N_{\lambda}}^{0}\right\}$ and center point $v_{0}$. Consider also the hyper-rectangles $X_{0}^{j} \subset X_{0}, j=1,2, \ldots, N_{0}$ with vertices respectively $\Lambda^{j}=\left\{\lambda_{1}^{j}, \lambda_{2}^{j}, \ldots, \lambda_{N_{\lambda}}^{j}\right\}, j=1,2, \ldots, N_{0}$. Suppose $X_{0}^{1} \subset X_{0}^{2} \subset \ldots \subset X_{0}^{N_{0}}$. For each of the hyper-rectangles $X_{0}$ and $X_{0}^{j} \subset X_{0}$, $j=1,2, \ldots, N_{0}$, denote the set of its facets centers with $\Phi^{j}=\left\{\phi_{1}^{j}, \phi_{2}^{j}, \ldots, \phi_{N_{\phi}}^{j}\right\}$, $j=0,1,2, \ldots, N_{0}$. Define the set of all points $V_{0}=\left\{v_{0}, v_{1}, v_{2}, \ldots, v_{N_{1}}\right\}$, where $v_{i} \in\left\{\bigcup_{j=0}^{N_{0}} \Lambda^{j}\right\} \bigcup\left\{\bigcup_{j=0}^{N_{0}} \Phi^{j}\right\}, i=1,2, \ldots, N_{1}$.

A close-to-global solution $U^{*}\left(v_{i}\right)$ of problem P2 at a point $v_{i} \in V_{0}$ is computed by using the routine 'glcSolve' of the TOMLAB optimization environment in Matlab [4]. The routine 'glcSolve' implements an extended version of the DIRECT algorithm [5], that handles problems with both nonlinear and integer

Int. Workshop on Assessment and Future Directions of NMPC

Pavia, Italy, September 5-9, 2008 
constraints. The DIRECT algorithm (DIviding RECTangles) [5] is a deterministic sampling algorithm for searching for the global minimum of a multivariate function subject to constraints, using no derivative information. It is a modification of the standard Lipschitzian approach that eliminates the need to specify a Lipschitz constant.

Based on the close-to-global solutions $U^{*}\left(v_{i}\right)$ at all points $v_{i} \in V_{0}$, a local constant approximation $\widehat{U}_{0}(\tilde{x})=K_{0}$ to the optimal solution $U^{*}(\tilde{x})$, feasible on the set $V_{0}$ and valid in the whole hyper-rectangle $X_{0}$, is determined by applying the following procedure:

Procedure 2 (Computation of explicit approximate solution):

Consider any hyper-rectangle $X_{0} \subseteq X$ with a set of points $V_{0}=\left\{v_{0}, v_{1}, \ldots, v_{N_{1}}\right\}$ determined by applying Procedure 1 . Compute $K_{0}$ by solving the following NIP:

$$
\min _{K_{0} \in U^{B}} \sum_{i=0}^{N_{1}}\left(J\left(K_{0}, v_{i}\right)-V^{*}\left(v_{i}\right)\right) \quad \text { subject to } G\left(K_{0}, v_{i}\right) \leq 0, \forall v_{i} \in V_{0}
$$

\subsection{Estimation of error bounds}

Suppose that a constant function $\widehat{U}_{0}(\tilde{x})=K_{0}$ that is feasible on $V_{0} \subset X_{0}$ has been determined by applying Procedure 2. Then, for the cost function approximation error in $X_{0}$ we have:

$$
\varepsilon(\tilde{x})=\widehat{V}(\tilde{x})-V^{*}(\tilde{x}) \leq \varepsilon_{0}, \quad \tilde{x} \in X_{0}
$$

where $\widehat{V}(\tilde{x})=J\left(\widehat{U}_{0}(\tilde{x}), \tilde{x}\right)$ is the sub-optimal cost and $V^{*}(\tilde{x})$ denotes the cost corresponding to the close-to-global solution $U^{*}(\tilde{x})$, i.e. $V^{*}(\tilde{x})=J\left(U^{*}(\tilde{x}), \tilde{x}\right)$. The following procedure can be used to obtain an estimate $\widehat{\varepsilon}_{0}$ of the maximal approximation error $\varepsilon_{0}$ in $X_{0}$.

Procedure 3 (Computation of error bound approximation):

Consider any hyper-rectangle $X_{0} \subseteq X$ with a set of points $V_{0}=\left\{v_{0}, v_{1}, \ldots, v_{N_{1}}\right\}$ determined by applying Procedure 1. Compute an estimate $\widehat{\varepsilon}_{0}$ of the error bound $\varepsilon_{0}$ through the following maximization:

$$
\widehat{\varepsilon}_{0}=\max _{i \in\left\{0,1,2, \ldots, N_{1}\right\}}\left(\widehat{V}\left(v_{i}\right)-V^{*}\left(v_{i}\right)\right)
$$

\subsection{Approximate mp-NIP algorithm}

Assume the tolerance $\bar{\varepsilon}>0$ of the cost function approximation error is given. The following algorithm is proposed to design explicit reference tracking quantized NMPC:

Algorithm 1 (explicit reference tracking quantized NMPC)

1. Initialize the partition to the whole hyper-rectangle, i.e. $\Pi=\{X\}$. Mark the hyper-rectangle $X$ as unexplored.

2. Select any unexplored hyper-rectangle $X_{0} \in \Pi$. If no such hyperrectangle exists, terminate.

3. Generate a set of points $V_{0}=\left\{v_{0}, v_{1}, v_{2}, \ldots, v_{N_{1}}\right\}$ associated to $X_{0}$ by applying Procedure 1.

4. Compute a solution to problem P2 for $\tilde{x}$ fixed to each of the points $v_{i}, i=0,1,2, \ldots, N_{1}$ by using routine 'glcSolve' of TOMLAB optimization environment. If problem $\mathrm{P} 2$ has a feasible solution at all these points, go to step

Int. Workshop on Assessment and Future Directions of NMPC

Pavia, Italy, September 5-9, 2008 
6. Otherwise, go to step 5 .

5. Compute the size of $X_{0}$ using some metric. If it is smaller than some given tolerance, mark $X_{0}$ infeasible and explored and go to step 2. Otherwise, split $X_{0}$ into hyper-rectangles $X_{1}, X_{2}, \ldots, X_{N_{s}}$ by applying the heuristic rule 1 from [3]. Mark $X_{1}, X_{2}, \ldots, X_{N_{s}}$ unexplored, remove $X_{0}$ from $\Pi$, add $X_{1}, X_{2}$, $\ldots, X_{N_{s}}$ to $\Pi$, and go to step 2 .

6. Compute a constant function $\widehat{U}_{0}(\tilde{x})$ using Procedure 2, as an approximation to be used in $X_{0}$. If no feasible solution was found, split $X_{0}$ into two hyper-rectangles $X_{1}$ and $X_{2}$ by applying the heuristic rule 3 from [3]. Mark $X_{1}$ and $X_{2}$ unexplored, remove $X_{0}$ from $\Pi$, add $X_{1}$ and $X_{2}$ to $\Pi$, and go to step 2.

7. Compute an estimate $\widehat{\varepsilon}_{0}$ of the error bound $\varepsilon_{0}$ in $X_{0}$ by applying Procedure 3. If $\widehat{\varepsilon}_{0} \leq \bar{\varepsilon}$, mark $X_{0}$ as explored and feasible and go to step 2. Otherwise, split $X_{0}$ into two hyper-rectangles $X_{1}$ and $X_{2}$ by applying Procedure 4 from [3]. Mark $X_{1}$ and $X_{2}$ unexplored, remove $X_{0}$ from $\Pi$, add $X_{1}$ and $X_{2}$ to $\Pi$, and go to step 2 .

\section{Explicit quantized NMPC of an electropneu- matic clutch actuator using on/off valves}

Here, a pneumatic actuator of an electropneumatic clutch system is considered. The pneumatic actuator acts on the clutch plates through the clutch spring, and the state of the clutch directly depends on the actuator position. The actuator is controlled by using on/off valves. In comparison to proportional valves, the on/off valves are smaller and cheaper. In [7], [9] the case when only fully open and closed are possible states of the valves is considered. Then, a controller is designed to govern switches between these states based on backstepping and Lyapunov theory. It should be noted however, that the methods in [7], [9] can not explicitly handle the constraints imposed on the clutch actuator position. On the other hand, Model Predictive Control (MPC) is an optimization based method for control which can explicitly handle both state and input constraints. This makes the MPC methodology very suitable to the optimal control of the clutch actuator. The fast dynamics of the clutch actuator, characterized with sampling time of about $0.01[\mathrm{~s}]$ requires the design of an explicit MPC controller, where the only computation performed on-line would be a simple function evaluation.

\subsection{Description of the electropneumatic clutch actuator}

The clutch actuator system is shown in Figure 1. To control both supply to and exhaust from the clutch actuator chamber, at least one pair of on/off valves are needed. As we only allow these to be fully open or closed, with two valves and under the assumption of choked flow, we restrict the flow of the clutch actuator to three possible values: maximum flow into the volume, maximum flow out of the volume, or no flow [7], [9]. The electronic control unit (ECU) calculates and sets voltage signals to control the on/off valves. These signals control whether the valve should open or close, and thus also the flow into the actuator. A position sensor measures position and feeds it back to the ECU. To calculate the control signals, knowledge of other states of the system are also needed, and these can be obtained either by sensors or by estimation.

Int. Workshop on Assessment and Future Directions of NMPC

Pavia, Italy, September 5-9, 2008 


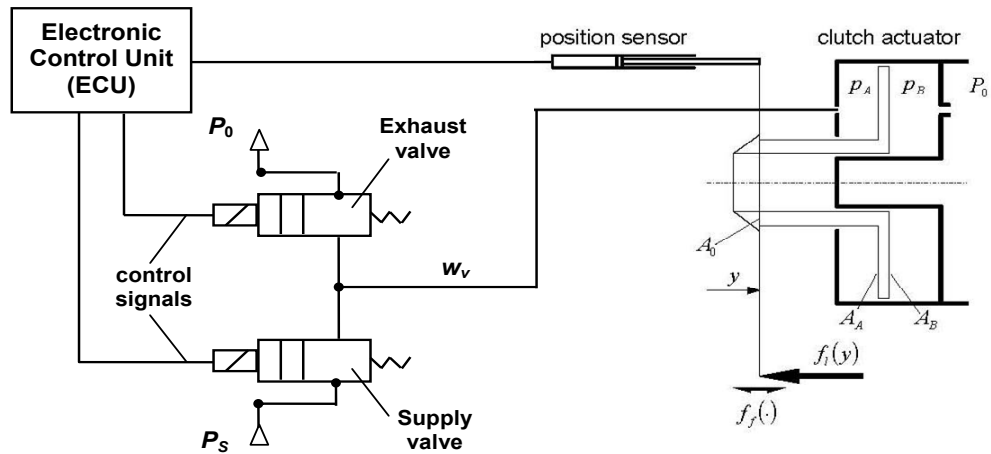

Figure 1: Electropneumatic clutch actuator ([6], [9]).

The full 5-th order model of the clutch actuator dynamics is the following [6]:

$$
\begin{aligned}
& \dot{y}=v \\
& \dot{v}=\frac{1}{M}\left(A_{0} P_{0}+A_{A} p_{A}-A_{B} p_{B}-f_{f}(v, z)-f_{l}(y)\right) \\
& \dot{p}_{A}=-\frac{A_{A}}{V_{A}(y)} v p_{A}+\frac{R T_{0}}{V_{A}(y)} w_{v}\left(p_{A}, u\right) \\
& \dot{p}_{B}=\frac{A_{B}}{V_{B}(y)} v p_{B}+\frac{R T_{0}}{V_{B}(y)} w_{r}\left(p_{B}\right) \\
& \dot{z}=v-\frac{K_{z}}{F_{C}}|v|_{q} z
\end{aligned}
$$

where $y$ is the position, $v$ is the velocity, $p_{A}$ is the pressure in chamber $\mathrm{A}, p_{B}$ is the pressure in chamber $\mathrm{B}, z$ is the friction state, $w_{v}\left(p_{A}, u\right)$ is the flow to/from chamber $\mathrm{A}, w_{r}\left(p_{B}\right)$ is the flow to/from chamber $\mathrm{B}, u$ is an integer control variable introduced below, and $V_{A}(y)$ and $V_{B}(y)$ are the volumes of chambers $\mathrm{A}$ and $\mathrm{B}$. The meaning of the parameters is the following: $A_{A}$ and $A_{B}$ are the areas of chambers $\mathrm{A}$ and $\mathrm{B}, A_{0}=A_{B}-A_{A}$ is piston area, $M$ is piston mass, $P_{0}$ is the ambient pressure, $T_{0}$ is the temperature, $R$ is the gas constant of air, $K_{z}$ is asperity stiffness, $F_{C}$ is Coulomb friction. In $(20),|v|_{q}=\sqrt{v^{2}+\sigma^{2}}$, where $\sigma>0$ is an arbitrary small design parameter. In (17), $f_{l}(y)$ and $f_{f}(v, z)$ are the clutch load and the friction force, described by:

$$
f_{l}(y)=K_{l}\left(1-e^{-L_{l} y}\right)-M_{l} y, f_{f}(v, z)=D_{v} v+K_{z} z+D_{\dot{z}} \dot{z}(v, z)
$$

An integer control variable $u \in U^{A}=\{1,2,3\}$ is introduced which is related to the flow $w_{v}\left(p_{A}, u\right)$ to/from chamber $\mathrm{A}$ in the following way:

$$
\begin{aligned}
& u=1 \Rightarrow w_{v}\left(p_{A}, 1\right)=-\rho_{0} C_{v, \text { out }} \psi\left(r, B_{v, \text { out }}\right) p_{A}, \quad r=\frac{P_{0}}{p_{A}} \\
& u=2 \Rightarrow w_{v}\left(p_{A}, 2\right)=0 \\
& u=3 \Rightarrow w_{v}\left(p_{A}, 3\right)=\rho_{0} C_{v, \text { in }} \psi\left(r, B_{v, \text { in }}\right) P_{S}, \quad r=\frac{p_{A}}{P_{S}}
\end{aligned}
$$

In $(24), P_{S}$ is the supply pressure. Therefore, $u=1$ corresponds to maximal flow from chamber $\mathrm{A}, u=2$ means no flow, and $u=3$ corresponds to maximal flow to chamber A. The expressions for the valve flow function $\psi\left(r, B_{v, \text { in } / \text { out }}\right)$, as well as for the flow $w_{r}\left(p_{B}\right)$ to/from chamber B can be found in [6]. 


\subsection{Design of explicit quantized NMPC}

In order to reduce the computational burden, the design of the explicit quantized NMPC controller is based on a simplified 3-rd order model of the clutch actuator, where the states are the actuator position $y^{s}$, the velocity $v^{s}$ and the pressure $p_{A}^{s}$ in chamber A:

$$
\begin{aligned}
& \dot{y}^{s}=v^{s} \\
& \dot{v}^{s}=\frac{1}{M}\left(-A_{A} P_{0}+A_{A} p_{A}^{s}-f_{f}^{*}\left(v^{s}\right)-f_{l}\left(y^{s}\right)\right) \\
& \dot{p}_{A}^{s}=-\frac{A_{A}}{V_{A}\left(y^{s}\right)} v^{s} p_{A}^{s}+\frac{R T_{0}}{V_{A}\left(y^{s}\right)} w_{v^{s}}\left(p_{A}^{s}, u\right)
\end{aligned}
$$

In $(26), f_{f}^{*}\left(v^{s}\right)=D_{v} v^{s}+F_{C} \frac{v^{s}}{\sqrt{v^{s}+\sigma^{2}}}$ is a static sliding friction characteristic [6]. The system (25)-(27) is discretized using a sampling time $T_{s}=0.01[\mathrm{~s}]$ and zero-order hold (the control input is assumed constant over the sampling interval). The forward Euler method with stepsize $T_{E}=0.0001[\mathrm{~s}]$ is used to integrate the equations (25)-(27). The control objective is to have the actuator position $y^{s}$ track a reference signal $r(t)>0$, which is achieved by minimizing the following cost function:

$$
\begin{aligned}
J\left(U, y^{s}(t), r(t)\right)= & \sum_{k=0}^{N-1}\left[Q\left(\frac{y_{t+k \mid t}^{s}-r(t)}{r(t)}\right)^{2}+R\left(\frac{w_{v^{s}}\left(p_{A, t+k \mid t}^{s}, u_{t+k}\right)}{w_{v^{s}, \max }-w_{v^{s}, \min }}\right)^{2}\right] \\
& +P\left(\frac{y_{t+N \mid t}^{s}-r(t)}{r(t)}\right)^{2}
\end{aligned}
$$

where $N=10$ is the horizon, $Q=1, R=0.1, P=1$ are the weighting coefficients, and $w_{v^{s}, \max }$ and $w_{v^{s}, \text { min }}$ are the maximal and the minimal flows to/from chamber A. The following constraints are imposed:

$y_{\text {min }} \leq y_{t+k \mid t}^{s} \leq y_{\max }, k=1, \ldots, N ; u_{t+k} \in U^{A}=\{1,2,3\}, k=0,1, \ldots, N-1$

where $y_{\min }=0, y_{\max }=0.025[\mathrm{~m}]$. In $(28), U \in U^{B}=\left(U^{A}\right)^{N}$. The quantized NMPC minimizes the cost (28) subject to the system equations $(25)-(27)$ and the constraints (29). The extended state vector is $\tilde{x}(t)=\left[e(t), v^{s}(t), p_{A}^{s}(t), r(t)\right] \in$ $\mathbb{R}^{4}$, where the state $e(t)$ is the projected reference tracking error defined as:

$$
e(t)=\left\{\begin{array}{l}
r(t)-y^{s}(t), \text { if }-0.005 \leq r(t)-y^{s}(t) \leq 0.005 \\
-0.005, \text { if } r(t)-y^{s}(t)<-0.005 \\
0.005, \text { if } r(t)-y^{s}(t)>0.005
\end{array}\right.
$$

The state space to be partitioned is 4-dimensional and it is defined by $X=$ $[-0.005 ; 0.005] \times[-0.05 ; 0.15] \times\left[P_{0} ; P_{S}\right] \times[0.0001 ; 0.024]$. The cost function approximation tolerance is chosen as $\bar{\varepsilon}\left(X_{0}\right)=\max \left(\bar{\varepsilon}_{a}, \bar{\varepsilon}_{r} \min _{\tilde{x} \in X_{0}} V^{*}(\tilde{x})\right)$, where $\bar{\varepsilon}_{a}=0.001$ and $\bar{\varepsilon}_{r}=0.02$ are the absolute and the relative tolerances. The partition has 10871 regions and 17 levels of search. Thus, 17 arithmetic operations are needed in real-time to compute the control input (17 comparisons).

The performance of the explicit quantized NMPC controller was simulated for a typical clutch reference signal and the resulting response is depicted in Figure 2. The simulations are based on the full 5-th order model (16)-(20).

Int. Workshop on Assessment and Future Directions of NMPC 

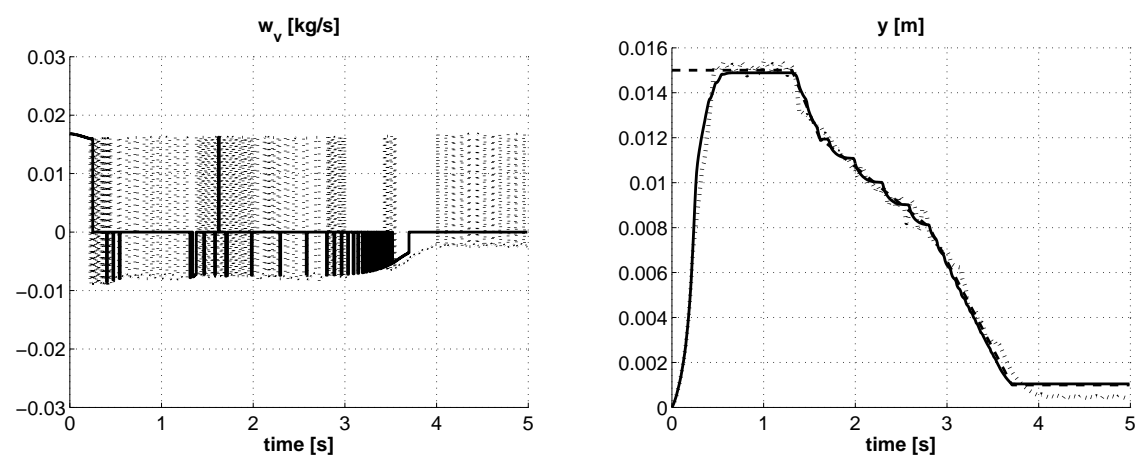

Figure 2: Left: The valve flow $w_{v}\left(p_{A}, u\right)$. Right: The clutch actuator position $y$. The dotted curves are with the approximate explicit quantized NMPC, the solid curves are with the exact quantized NMPC and the dashed curve is the reference signal.

Acknowledgement This work was sponsored by the Research Council of Norway, projects 168194/I40 and 177616/V30.

\section{References}

[1] A. Bemporad. Multiparametric nonlinear integer programming and explicit quantized optimal control. 42-nd IEEE Conference an Decision and Control, Maui, Hawaii, USA, December 2003.

[2] A.V. Fiacco. Introduction to sensitivity and stability analysis in nonlinear programming. Academic Press, Orlando, Florida, 1983.

[3] A. Grancharova, T.A. Johansen, and P. Tøndel. Computational aspects of approximate explicit nonlinear model predictive control. In R. Findeisen, F. Allgöwer and L. Biegler, editors, Assessment and Future Directions of Nonlinear Model Predictive Control, Lecture Notes in Control and Information Sciences, volume 358, pages 181-192. Springer-Verlag, Berlin/Heidelberg, 2007.

[4] K. Holmström, A.O. Göran, and M.M. Edvall. User's guide for TOMLAB, April, 2007.

[5] D.R. Jones. The DIRECT global optimization algorithm. In C.A. Floudas and P.M. Pardalos, editors, Encyclopedia of optimization, volume 1, pages 431-440. Kluwer, Dordrecht, 2001.

[6] G.O. Kaasa. Nonlinear output-feedback control applied to electro-pneumatic clutch actuation in heavy-duty trucks. Ph.D. Thesis, Norwegian University of Science and Technology, 2006.

[7] H. Langjord, T.A. Johansen, and J.P. Hespanha. Switched control of an electropneumatic clutch actuator using on/off valves. American Control Conference, Seattle, 2008.

[8] B. Picasso, S. Pancanti, A. Bemporad, and A. Bicchi. Receding-horizon control of LTI systems with quantized inputs. IFAC Conference on Analysis and Design of Hybrid Systems, Saint Malo, France, June 2002.

[9] H. Sande, T.A. Johansen, G.O. Kaasa, S.R. Snare, and C. Bratli. Switched backstepping control of an electropneumatic clutch actuator using on/off valves. American Control Conference, New York, 2007. 